\title{
Nanoscale dopant-induced dots and potential fluctuations in GaAs
}

\author{
N. D. Jäger and K. Urban \\ Institut für Festkörperforschung, Forschungszentrum Jülich GmbH, 52425 Jülich, Germany \\ E. R. Weber \\ Department of Materials Sciences, University of California, and Materials Science Division, Lawrence \\ Berkeley National Laboratory, Berkeley, California 94720 \\ Ph. Ebert ${ }^{\mathrm{a})}$ \\ Institut für Festkörperforschung, Forschungszentrum Jülich GmbH, 52425 Jülich, Germany
}

(Received 20 January 2003; accepted 26 February 2003)

\begin{abstract}
We identified $p$-type nanoscale dopant-induced dots that are formed by fluctuations of the dopant atom distribution in sufficiently thin GaAs $p-n$ multilayers. Their electronic structure and the resulting potential variations were investigated by cross-sectional scanning tunneling microscopy and spectroscopy as a function of the number of dopant atoms within the dot. We find significant changes in the current-voltage characteristics of the dots compared to spatially nonconfined material, due to a reduced ability to screen the tip's electric field. This indicates a limited ability to deplete the dots of free holes arising from the presence of confining potentials surrounding the dopant-induced dots. (C) 2003 American Institute of Physics. [DOI: 10.1063/1.1569419]
\end{abstract}

State-of-the-art semiconductor devices currently consist of structures with dimensions as small as $70 \mathrm{~nm}$ defined by the gate length of the transistors. Research is focusing on a further reduction to $20-30 \mathrm{~nm}$ aimed for production in the year 2007. ${ }^{1}$ At such small dimensions the electrical potential distribution within the devices cannot be treated as continuous anymore. The potential is rather governed by individual, discrete ionized dopant atoms. Consequently, statistical fluctuations in dopant distribution limit the achievable miniaturization of semiconductor devices, by introducing "intrinsic" variations in individual device characteristics, such as turn on voltage and noise level spectra. ${ }^{2,3}$ However, it has turned out that a direct experimental access to individual dopant atoms and the corresponding potential distributions is a very difficult task. ${ }^{4,5}$

This letter presents a direct experimental proof of local variations of electronic characteristics and potential induced by fluctuations of the dopant atom distribution on the tens of nanometer scale. In $n-p-n$ GaAs layer structures the fluctuations lead to localized nanoscale dopant-induced dots spatially confined by potential barriers. Using cross-sectional scanning tunneling microscopy (STM) and spectroscopy ${ }^{6,7}$ we identify such dots and investigate their electronic structure as a function of the number of dopant atoms within the dot. We demonstrate that the properties of the dots change drastically due to the spatial confinement of the free charge carriers even at room temperature.

The structure investigated consisted of alternating 30nm-thick $p$ - and $n$-doped layers grown by molecular beam epitaxy (MBE) on a (001) oriented GaAs substrate, with C and $\mathrm{Si}$ dopant concentrations of $(5 \pm 1) \times 10^{18} \mathrm{~cm}^{-3}$ and $(4 \pm 1) \times 10^{18} \mathrm{~cm}^{-3}$, respectively. The sample was cleaved in ultrahigh vacuum to expose a (110) cross-sectional view of the layers. This view was imaged by STM in the

${ }^{\text {a)} E l e c t r o n i c ~ m a i l: ~ p . e b e r t @ f z-j u e l i c h . d e ~}$ constant-current mode with atomic resolution at room temperature.

Figure 1 shows a sequence of several $n$ - and $p$-doped layers, which we identified on basis of the growth sequence and current-voltage spectra. ${ }^{8}$ At the interface between $n$ and $p$-doped layers the presence of a depletion zone gives rise in STM to a dark line. ${ }^{9}$ The bright hillocks are signatures of the individual dopant atoms. For the present work the $p$-doped layer on the right-hand side of Fig. 1 is of particular interest. It exhibits several encircled areas labeled $d_{2}, d_{4}$, and $d_{14}$ according to the number of dopant atoms visible within the area. These areas are bordered along the growth direction by $n$-doped layers and perpendicular to the growth direction by zones with no dopant atoms (dark contrast areas labeled $d_{0}$ ). These zones with no dopant atoms exhibit tunneling spectra (Fig. 2) with typical characteristics of a depleted region (as discussed later). Thus, the areas $\left(d_{2}, d_{4}\right.$, and $\left.d_{14}\right)$ are semiconductor dots, whose confining potential for free holes is defined by the doping of the surrounding and thus by built-in potentials in the order of a few tenths of electron volts (border toward depleted zones) to $1.4 \mathrm{eV}$ (toward $n$-doped layer).

During acquisition of the constant-current STM images we also measured current-voltage spectra at the different encircled areas in Fig. 1. In order to allow a proper comparison of the spectra in Fig. 2, we normalized the spectra to a common tip-sample separation. ${ }^{9}$ All spectra are essentially identical at positive sample voltages. In contrast, at negative sample voltages the current-voltage curves are shifted relative to each other. The fewer dopants are inside the cluster, the greater is the shift towards more negative voltages relative to the spectrum of the spatially extended $p$-doped layer (labeled $p$ in Fig. 1). The voltage shifts can be better illustrated by plotting the difference in sample voltage required for a fixed current as a function of the number of dopant atoms visible in a dot (Fig. 3). Figure 3 shows that at negative sample bias (VB shift) the shifts scale with the number 


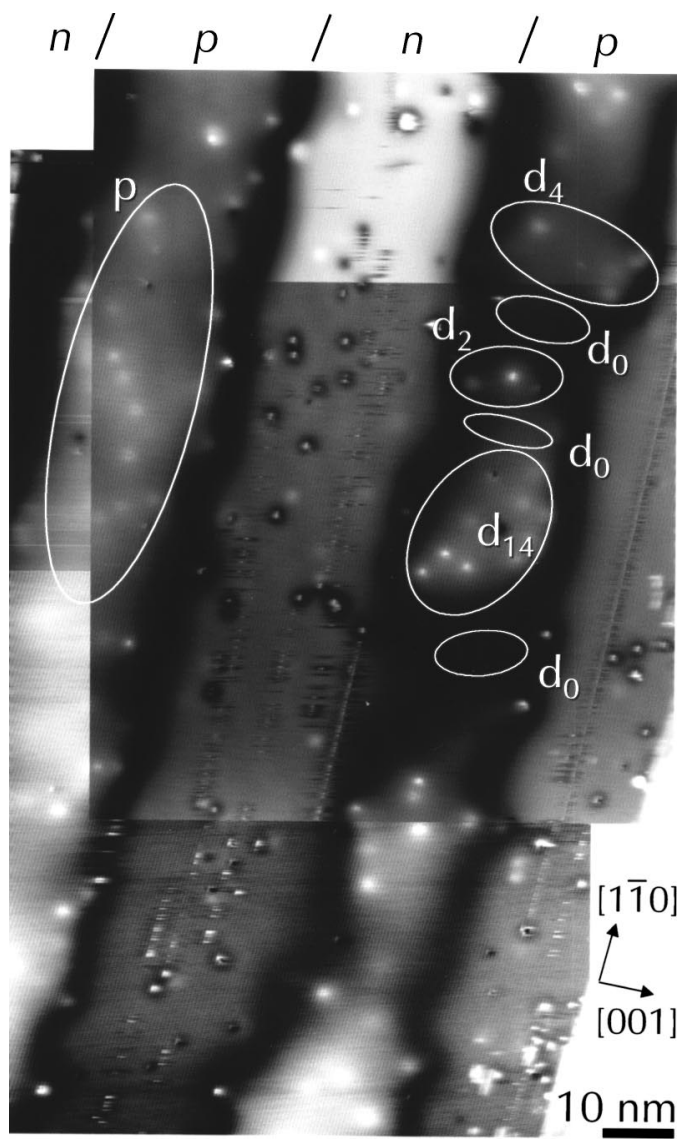

FIG. 1. Atomically resolved scanning tunneling microscopy image of several $p$ - and $n$-doped layers taken at $-2.5 \mathrm{~V}$ sample voltage and a current of $0.1 \mathrm{nA}$. The bright hillocks are signatures of dopant atoms. In the encircled areas the current voltage curves of Fig. 2 were recorded. The $p$-doped areas $d_{2}, d_{4}$, and $d_{14}$ are dopant-induced dots confined by potential barriers due to the doping of the surrounding areas ( $n$-type and lack of dopants). The growth direction is [001].

of dopant atoms in the dot, whereas at positive voltages no shift occurs (CB shift) for all set currents.

The local variation of the dopant concentration apparent in Fig. 1 is a result of the combination of statistical incorporation $^{1}$ and many-body effects in the repulsive screened Coulomb interactions between charged dopant atoms. ${ }^{7}$ These effects occur in the melt doped bulk $\mathrm{GaAs}^{7}$ as well as in thin MBE grown layers. ${ }^{8}$ In bulk GaAs or in suf-

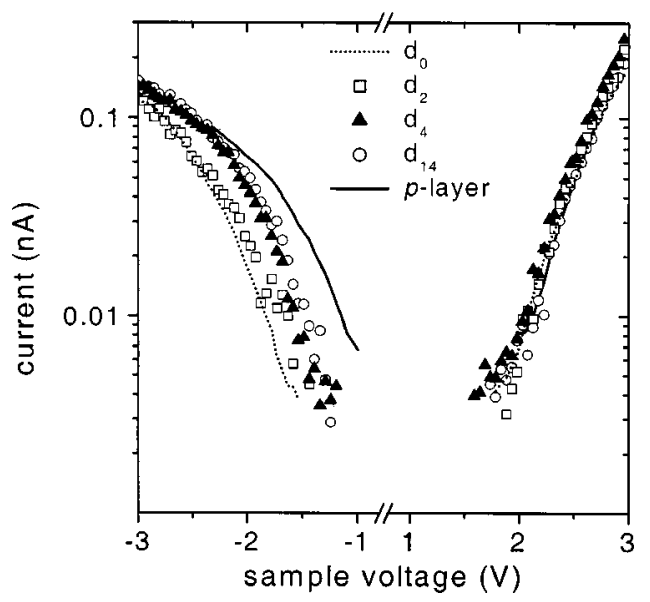

FIG. 2. Current-voltage curves for the different dopant-induced dots

the position of the valence band edge at the surface of our
marked in Fig. 1 .
Downloaded 21 Dec 2006 to 134.94.122.39. Redistribution subject to AIP license or copyright, see http://apl.aip.org/apl/copyright.jsp

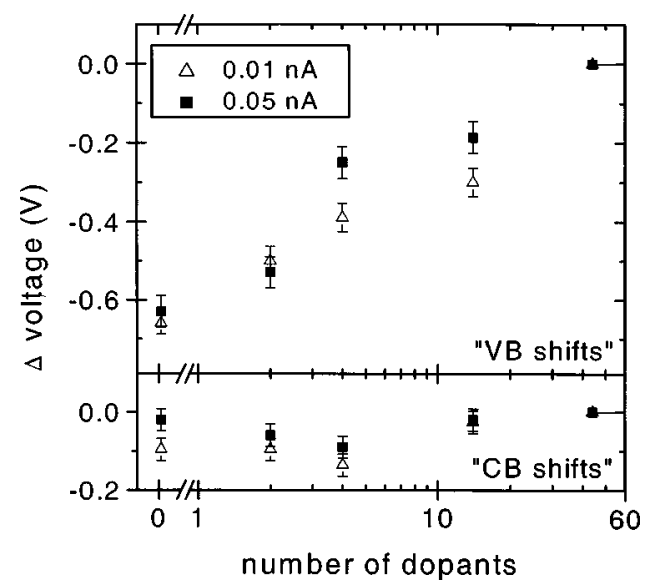

FIG. 3. Voltage differences relative to the spatially extended $p$-doped layer (labeled $p$ in Fig. 1) needed to obtain a tunneling current of 0.01 and 0.05 $\mathrm{nA}$ as a function of number of dopant atoms visible within the dots. The values were taken from Fig. 2 at negative sample voltages, (VB shifts), and positive sample voltages [CB shifts].

ficiently thick layers the fluctuations of the dopant concentration do not significantly affect the local Fermi level position. In thin $p$-doped layers sandwiched between $n$-doped areas, however, the electronic properties depend very sensitively on the exact dopant distribution governed by the individual statistical incorporation events: in our particular example this leads to one layer with nearly homogeneous electronic properties (marked $p$ in Fig. 1) and to another layer broken apart into electronically isolated dots $\left(d_{2}, d_{4}, d_{14}\right)$. The electronic separation of the dots within the thin $p$-doped layer is induced by the neighboring $n$-doped layers, which push down the valence band edge wherever $p$-dopant atoms are locally missing within the nominally $p$-doped layer. This explanation is supported by our tunneling spectra.

At close look the electronically homogeneous $p$-doped layer exhibits local fluctuations in the dopant atom distribution too, but the tunneling spectra do not show any measurable spatial variation. Thus, the bare presence of fluctuations in dopant concentration is not sufficient to explain the differences in the spectra of Fig. 2. Any effect of different Fermi levels in the different dots can be neglected, too, because the voltage shifts observed here are much too large for the variations of the dopant concentration between $2 \times 10^{18}$ and 4 $\times 10^{18} \mathrm{~cm}^{-3}$. Thus, the differences in the tunneling spectra can only be due to the electronic separation of the dots and the resulting confinement potential for the free holes within the dots.

For a quantitative discussion of the spectra, we recall that the spectra consist of the current from valence and into conduction band states at negative and positive voltages, respectively, with the band gap region in between. ${ }^{10,11}$ At a fixed voltage the tunneling current is determined by the energetic positions of the band edges underneath the tip:

At negative voltages, electrons tunnel from all filled valence band states lying between the valence band edge at the surface and the Fermi level of the tip. The size of this energy window is determined by the degree the tip bends the bands at the surface. Thus, the shifts of the spectra at negative voltages (Figs. 2 and 3) indicate that the tip pulls downward the position of the valence band edge at the surface of our AIP license or copyright, see http://apl.aip.org/apl/copyright.jsp 
dopant-induced dots the more, the less dopants are enclosed within the dot. This suggests a reduced screening ability of the dots with smaller numbers of dopant atoms.

The electric field of the tip is screened by negatively charged acceptors, whose free holes have to be pushed away. On this basis, a reduction in the ability to screen the field of the tip is due to a combination of (i) the impossibility to deplete the dopant-induced dots from the free holes and (ii) the number of acceptors within the dot.

(i) The first effect depends on the size of the dot. If the dimension of the dot is much larger than the depletion width induced by the screening of the tip's field, i.e., the field can be screened entirely by the acceptors within the dot, then the band bending is determined by the concentration of dopant atoms in the dot. As a consequence, the lower the dopant atom concentration, the larger is the voltage necessary to obtain a fixed tunnel current, ${ }^{12}$ because the tip-induced band bending increases. This effect is applicable to the spatially extended $p$-doped layer on the left hand side of Fig. 1, which is electronically homogeneous over dimensions of more than $100 \mathrm{~nm}$, which is much larger than the estimated depletion width of 10-25 $\mathrm{nm}$ for acceptor concentrations of 4 $\times 10^{18} \mathrm{~cm}^{-3}$ at $-2.5 \mathrm{~V}$ sample voltage.

If the dots' dimensions are similar to the width of the depletion zone, one also needs to consider whether the holes can actually be pushed away sufficiently to accommodate the screening of the tip's electric field. For the small dot sizes, this means that holes need to be pushed out of the enclosed dot areas to deplete them. We recall that our dots are confined by potential wells. These wells act as a barrier for the free holes and impedes the holes to be pushed out, such that the remaining negatively charged acceptors could screen the electric field of the tip. If the dot cannot be sufficiently depleted, the band bending increases, causing lower tunneling currents than expected for infinitely sized bulk GaAs crystals with the same dopant concentration. The effect of confinement of the free holes on the tunneling current becomes smaller the higher the applied voltage is, because the relative fraction of states inhibited to tunnel by the locally increased tip-induced band bending diminishes as more valence band states are involved in the tunneling process. In addition the depletion width is increasing with voltage and becomes eventually larger than the cluster size leading to an averaging of the characteristics of the locally different areas. These expectations are in agreement with the observation in Fig. 2.

At positive sample voltage no dependence of the current on the number of enclosed dopant atoms is found (Figs. 2 and 3 "CB shift"). This implies that the energetic position of the conduction band edge underneath the tip is the same for all areas investigated here. At positive sample voltage the electric field of the tip is screened by free holes accumulating at the $p$-doped surface. These holes feel no barrier to accumulate at the surface and thus can effectively screen the tip's field. Furthermore, the number of accumulated holes is determined by the density of valence band states, which is the same for all investigated areas, because the material is the same. This explains the almost invariant current-voltage spectra obtained on the various dots at positive sample voltages.

(ii) The earlier discussion also shows, that the ability to screen the electric field of the tip is directly proportional to the number of acceptors available within the electronically isolated dot. Thus, the fewer acceptors within the dot, the larger the tip-induced band bending and the larger the voltage shift observed in the tunneling spectra. This explanation is in agreement with our observations.

In conclusion, we identified and characterized dopantinduced dots in GaAs $p-n$ multilayers by scanning tunneling spectroscopy. We showed that already at room temperature the properties of the dots are determined by the presence of confining potentials surrounding the dots. The confinement leads at negative sample voltage to a reduced ability to screen the electric field of the tip compared to spatially nonconfined material, because of the limited depletion of the dots of free holes. For positive sample voltages no changes were found, because holes can freely accumulate at the surface underneath the tip. The similarity of the dots' configuration with $n-p-n$ transistors suggests that the physical mechanisms found here will also affect future miniaturized semiconductor devices, once they reach dimensions as small as the fluctuations of the dopant concentration.

The authors thank W. K. Liu from IQE Inc. for providing the sample and the Deutsche Forschungsgemeinschaft under Grant No. EB 197/2-1 as well as the Director, Office of Energy Research, Office of Basic Energy Research, Materials Science Division, US. Department of Energy under Contract No. DE-AC03-76SF00098 for financial support.

${ }^{1}$ International Technology Roadmap for Semiconductors (ITRS), 2001 ed. (Semiconductor Industry Association, San Jose, CA, 2001).

${ }^{2}$ J. D. Meindl, Q. Chen, and J. A. Davis, Science 293, 2044 (2001).

${ }^{3}$ A. Asenov, Nanotechnology 10, 153 (1999).

${ }^{4}$ W. D. Rau, P. Schwander, F. H. Baumann, W. Höppner, and A. Ourmazd, Phys. Rev. Lett. 82, 2614 (1999).

${ }^{5}$ P. M. Voyles, D. A. Muller, J. L. Grazul, P. H. Citrin, and H.-J. L. Grossmann, Nature (London) 416, 826 (2002).

${ }^{6}$ N. D. Jäger, E. R. Weber, and M. Salmeron, in: Microprobe Characterization of Optoelectronic Materials, edited by J. Jimenez (Taylor and Francis, London, 2002).

${ }^{7} \mathrm{Ph}$. Ebert, T. Zhang, F. Kluge, M. Simon, Z. Zhang, and K. Urban, Phys. Rev. Lett. 83, 757 (1999).

${ }^{8}$ N. D. Jäger, K. Urban, E. R. Weber, and Ph. Ebert, Phys. Rev. B 65, 235302 (2002).

${ }^{9}$ N. D. Jäger, M. Marso, M. Salmeron, E. R. Weber, K. Urban, and Ph. Ebert, Phys. Rev. B (in press).

${ }^{10}$ R. M. Feenstra and J. A. Stroscio, J. Vac. Sci. Technol. B 5, 923 (1987).

${ }^{11}$ R. M. Feenstra, J. A. Stroscio, J. Tersoff, and A. P. Fein, Phys. Rev. Lett. 58, 1192 (1987).

${ }^{12}$ R. Maboudian, K. Pond, V. Bressler-Hill, M. Wassermeier, P. M. Petroff, G. A. D. Briggs, and W. H. Weinberg, Surf. Sci. Lett. 275, L662 (1992). The measurements on $n$-doped GaAs show a reduced current with decreasing dopant concentration at a fixed positive voltage. For $p$-doped GaAs the same effects occur only at negative voltages. 